\title{
EEG spectra in twins: Evidence for a neglected mechanism of genetic determination
}

\author{
D. T. LYKKEN, A. TELLEGEN, and W. G. IACONO \\ University of Minnesota, Minneapolis, Minnesota
}

\begin{abstract}
Electroencephalographic (EEG) spectra obtained from monozygotic (MZ) twins show striking within-pair similarity, while spectra of dizygotic (DZ) twins are no more similar than those obtained from pairs of unrelated persons. At least one parameter of the EEG spectrum, the midfrequency of the alpha rhythm, is strongly correlated (.8) within pairs of $\mathrm{MZ}$ twins and yet its correlation within pairs of $\mathrm{DZ}$ twins is about zero. The frequency composition of the resting EEG may be an example of "emergenesis," in which the phenotypic character is determined by the interaction of independently heritable traits and is substantially altered if any genotypic component is changed. Such emergenic traits would not reveal their genetic origin in the usual analysis of pedigrees.
\end{abstract}

An electroencephalographic (EEG) spectrum is a graph of EEG voltage with respect to frequency. In our work, each spectrum consists of 200 ordinates which estimate the relative voltage at $.1-\mathrm{Hz}$ intervals, from .1 to $20.0 \mathrm{~Hz}$, contained in a 3-min sample of resting EEG, eyes closed. We have reported previously that EEG spectra of monozygotic (MZ) twins are remarkably similar when obtained at the same time and under similar conditions (Lykken, Tellegen, \& Thorkelson, 1974). This similarity, illustrated in Figure 1, can be measured by the spectrum difference ratio (SDR):

$$
S D R=\frac{D\left(A_{1}-B_{2}\right)+D\left(A_{2}-B_{1}\right)}{D\left(A_{1}-A_{2}\right)+D\left(B_{1}-B_{2}\right),}
$$

where, as shown in Figure 2, $A_{1}$ is the spectrum obtained from Twin A (the first-born) at the start of the session, $B_{2}$ is Twin B's spectrum at the end of the session, etc., and $D$ is the root-mean-squared difference between the two spectra specified within the parentheses.

When SDR $\leqslant 1.0$, one could say that, with respect to their EEG spectra, the twins resemble each other as much as they resemble themselves. SDRs for the 39 pairs of $\mathrm{MZ}$ twins previously reported are plotted in Figure 3A against a distribution of SDRs for pairs of unrelated persons (obtained by pairing each individual twin with all persons other than his/her cotwin); $33 \%$ of the $\mathrm{MZ}$ ratios are $\leqslant 1.0$, and the mean difference between the $\mathrm{MZ}$ and unrelated pairs distributions is highly significant $(\mathrm{p} \ll .001)$. We conclude that persons with identical genomes will usually

D. T. Lykken is in the Department of Psychiatry. His address is: Box 392, Mayo, University of Minnesota, Minneapolis, Minnesota 55455. A. Tellegen is with the Department of Psychology. W. G. Iacono is now with the Department of Psychology, University of British Columbia, Vancouver, B.C., Canada. display EEGs with nearly identical frequency composition.

If the EEG spectrum is genetically determined, the mechanism is undoubtedly polygenic. We have found, for example, that at least six orthogonal factors are required to account for some $80 \%$ of the common variance due to individual differences in EEG spectra (Lykken, 1975). While single-factor variables, such as stature, can be polygenic, multifactor variables are necessarily polygenic. When $\mathrm{MZ}$ twins are found to be very similar with respect to some polygenic trait, then, since they share on the average half their genes in common, the additive polygenic model predicts that dizygotic (DZ) twins will be at least half as similar as the MZs. An unexpected finding of the earlier study was that the DZ twins were no more similar in respect to their EEG spectra than the comparison sample of unrelated pairs. Figure 3B shows the distribution of SDRs for the 27 pairs of $\mathrm{DZ}$ twins in that study; the DZ sample mean is not significantly smaller than that for unrelated pairs.

We suggest that these findings illustrate an important phenomenon, neglected by behavior geneticists, that we propose to label emergenesis. At the genetic level, an emergenic trait can be defined as a phenotypic characteristic that results from the (nonadditive) interaction of a configuration of several independent genes. If all members of the gene pattern are present, the trait appears as an emergent phenomenon, not predictable as the simple sum of the effects of the individual genes separately assessed. An example of such an effect might be a chemical reaction the direction of which at each stage is determined by a different gene. The reaction product could be qualitatively different for each different gene pattern and would have emergent properties not inherent in the reacting constituents. At a molar level, an emergenic trait is defined as a characteristic that results from the interaction of two or more independent traits that are 


\section{MONOZYGOTIC TWINS Magnitude Spectra}
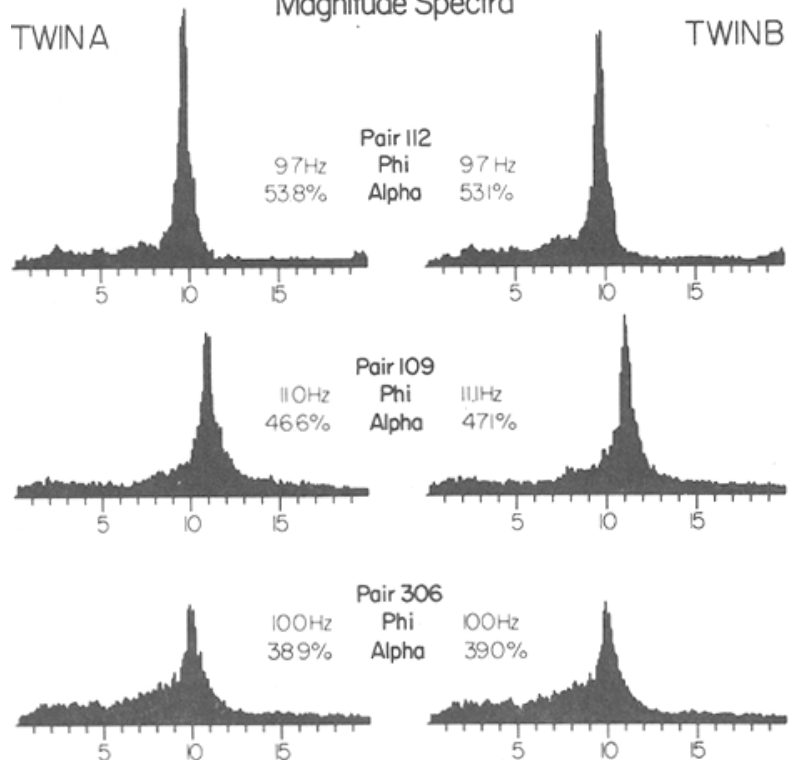

Pair 306

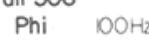

Alpho $390 \%$
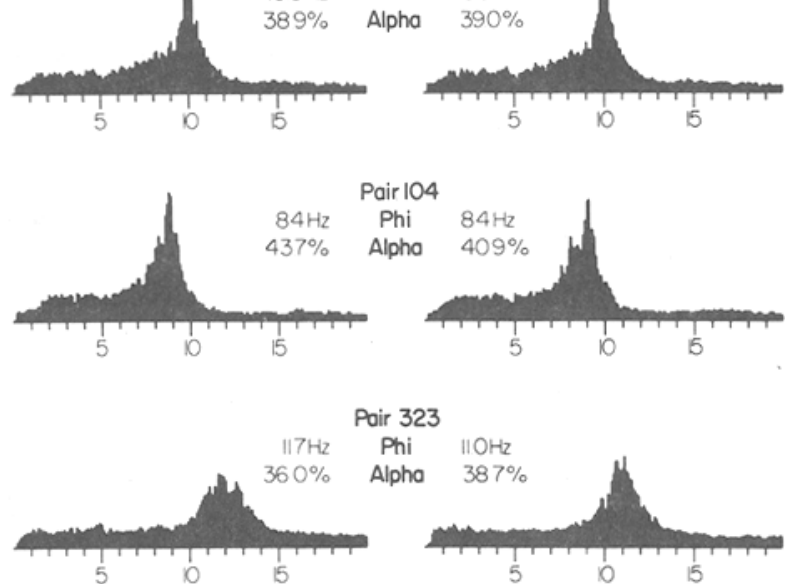

Poir 323
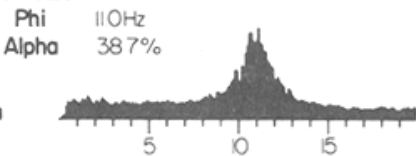

Pair 102

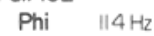

$194 \%$ Alpha $192 \%$
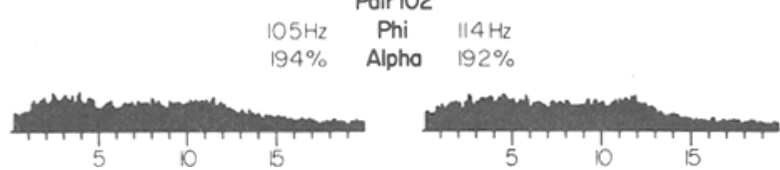

Figure 1. EEG spectra from 6 of the 39 monozygotic (MZ) twin pairs studied in 1974, selected to show the range of amount of alpha activity. Phi is the median frequency in a $3-\mathrm{Hz}$ band centered on the central peak. All spectra are standardized to unit area. (Reprinted from Lykken, Tellegen, \& Thorkelson, 1974.)

themselves in part genetically determined. $\mathrm{MZ}$ twins, because of their identical genomes, will show concordance for such emergenic traits. Because the components (genes or genotypic traits) of the pattern may vary independently, however, it is unlikely that $\mathrm{DZ}$ twins, having only about half their genes in common, would share all of the components of the pattern necessary for concordance (or similarity) in respect to emergenic traits. For the same reason, it would be unlikely for such traits to be passed from parents to offspring or to run in families.

This interpretation was first suggested to us by J. C. Loehlin, in 1974, in terms of the familiar ge- netic concept of epistasis. We agree, however, with Murphy's comment: “There is a perfectly respectable genetic phenomenon in which the effect of one gene masks that of another-'epistasis.' ... But the classical quantitative geneticist has acquired the deplorable habit of ascribing [all nonallelic interactions] to 'epistasis, etc.' .... What results is not science but devious accountancy"' (Murphy, 1979, p. 132). Epistasis,
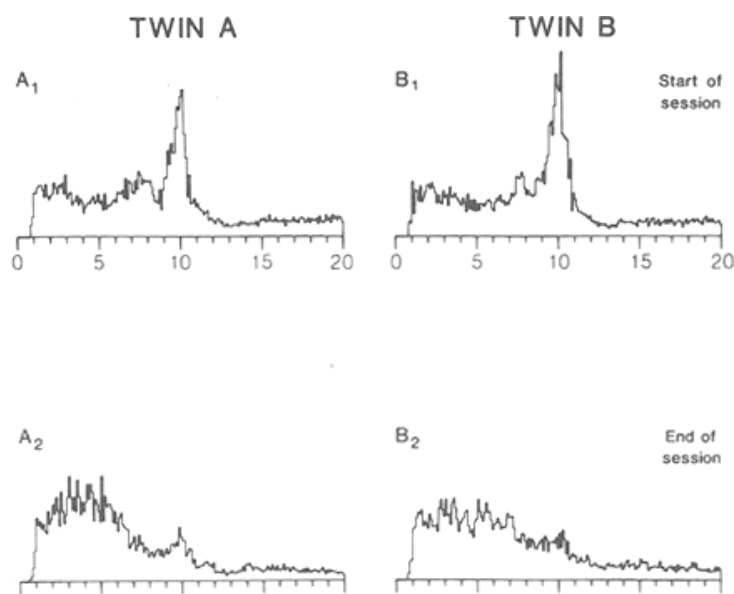

Figure 2. EEG magnitude spectra for one pair of monozygotic (MZ) twins. Each spectrum shows the relative activity from .5 to $20 \mathrm{~Hz}$ in a 3-min sample of EEG recorded between vertex and earlobe while the subject rested with eyes closed. These twins were unusual in that, although tested 3 weeks apart, both became drowsy by the end of the session and produced very similar changes in their EEG spectra.

\section{EEG SPECTRUM SIMILARITY OF TWINS TESTED TOGETHER: 1974 FINDINGS}

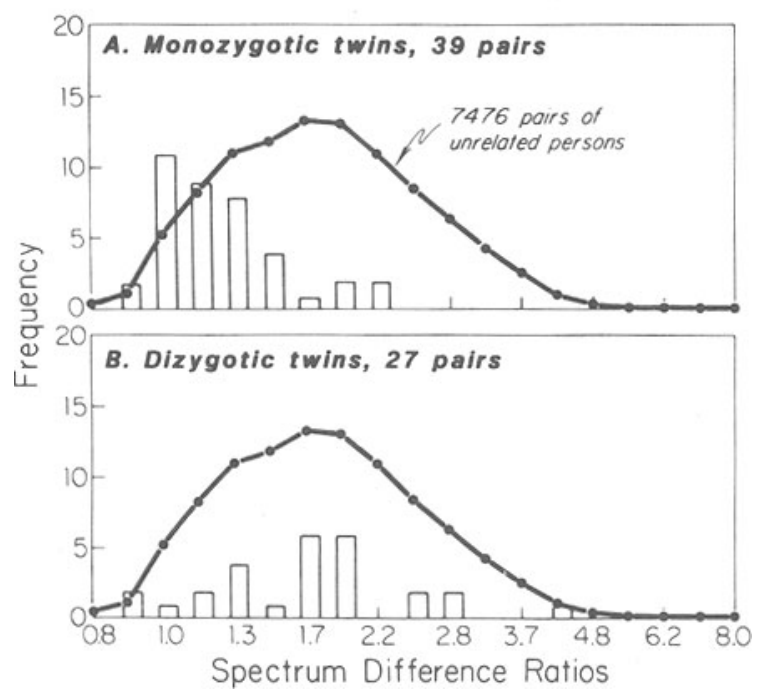

Figure 3. Distributions of spectrum difference ratios (SDRs) for MZ (A) and DZ (B) twins studied in 1974. The smooth curve is a comparison distribution of SDRs for unrelated pairs. When the SDR $\leqslant 1.0$, the paired spectra resemble each other as much as they resemble repeated spectra from the same individuals. While the spectra of $M Z$ twins are very similar, $D Z$ twin spectra are no more alike than those from pairs of unrelated persons. 
like dominance, is a special form of gene interaction (indeed, it is a "dominance" relation between two nonallelic genes), whereas the configural model invoked here is more general, a polygenic interaction in which each participant gene is essential to the outcome. We think Loehlin's concept is different and important enough to deserve its own term, and we propose the Greco-Latin hybrid emergenesis.

EEG spectra are influenced by the size and shape of the skull relative to the underlying tissues, by the details of cerebral anatomy, including the as yet unidentified components of the alpha generator, and especially by the microstructure associated with the subject's cognitive and emotional reactions in the testing situation. If spectra are very similar in persons with identical genomes, but not more similar in firstdegree relatives than in unrelated persons, the spectrum pattern may be an emergent consequence of the particular configuration of causal factors, each of which is itself genetically determined.

Another explanation for these findings might be that the effects of shared experience, usually assumed to enhance the within-pair similarity of twins, may actually decrease the similarity of DZ twins for some traits. The twins in the previous study came to the laboratory together and their EEG samples were obtained simultaneously. Since EEG spectra change with changes in the subject's mental state, the similarity observed for the MZ twins indicates that most MZ pairs were in similar states of arousal or that their mental states changed over time in similar ways, as illustrated in Figure 2. But perhaps the presence of the co-twin caused some twins to be in a mental state that was different from what they would have adopted had they come to the laboratory alone. This diverging tendency, if it did occur, would be expected to be stronger among $\mathrm{DZ}$ than among $\mathrm{MZ}$ pairs.

The least interesting, but possible, explanation of the previous findings is that they were due to errors of sampling or measurement against which conventional significance testing is no sure protection (Lykken, 1968). We report here a constructive replication in which spectra were obtained from a new sample of twins under somewhat modified circumstances.

\section{METHOD}

The 152 persons studied included $50 \mathrm{MZ}$ and 26 same-sex DZ pairs of adult twins ranging in age from 19 to 55, median 27 years. As is usual in work with adult volunteer twins (Lykken, Tellegen, \& DeRubeis, 1978), most of the pairs were female (46 of 76 pairs) and most pairs were monozygotic (50 of 76). Two EEG channels were recorded from electrodes at $\mathrm{C} 3$ and $\mathrm{C} 4$, both referenced against linked earlobes and providing bilateral frontoparietal EEG, in contrast to the single midline occipital $(\mathrm{Oz})$ channel used in the previous study. The subject sat alone in a shielded booth during the 1-h session, rather than side by side with his/her twin as in our earlier work. At the start, and again at the end of the session, the subject was asked to sit quietly in the dark, eyes closed, for $5 \mathrm{~min}$ of EEG recording. The two EEG channels were recorded on FM tape. The cleanest 3-min segment of each sample was later played back through a bandpass filter $(.5$ to $20 \mathrm{~Hz}, 24 \mathrm{~dB} /$ octave) into the A/D inputs of a PDP-12 computer which performed a fastFourier transform yielding a power spectrum with ordinate values at each $.1-\mathrm{Hz}$ increment from 0 to $20 \mathrm{~Hz}$. The square roots of these 200 values gave magnitude spectra which were then converted to unit areas.

\section{RESULTS}

Spectrum difference ratios were calculated for all twin pairs, Formula 1 being modified to accommodate two simultaneous spectra. A comparison distribution of unrelated pairs was obtained by computing SDRs between all unrelated pairs of individuals in the sample, a total of 9,940 pairs. As can be seen in Figure $4 \mathrm{C}$, the previous results for $\mathrm{MZ}$ twins were replicated. Even when obtained at different times on different days, the EEG spectra of MZ twins are very similar: $34 \%$ of the MZ SDRs are $\leqslant 1.0$ and the difference between the MZ and the UR means is highly significant $(\mathrm{p} \ll .001)$. Figure 4D shows that, as in the previous study, the spectra of DZ twins were no more alike than those for pairs of unrelated persons, even though, this time, the individual twins were brought to the laboratory separately. As can be seen in Figures 3 and 4, a few MZ pairs in both studies showed EEG spectra as dissimilar as the average unrelated pair. This fact does not refute the emergenic interpretation since, of course, no sample of EEG activity will be entirely determined by the genome; as illustrated in Figure 2, even the same individual may show considerable spectrum change from one time of testing to another.

To supplement the gross comparison provided by the spectrum difference ratio, one can also examine

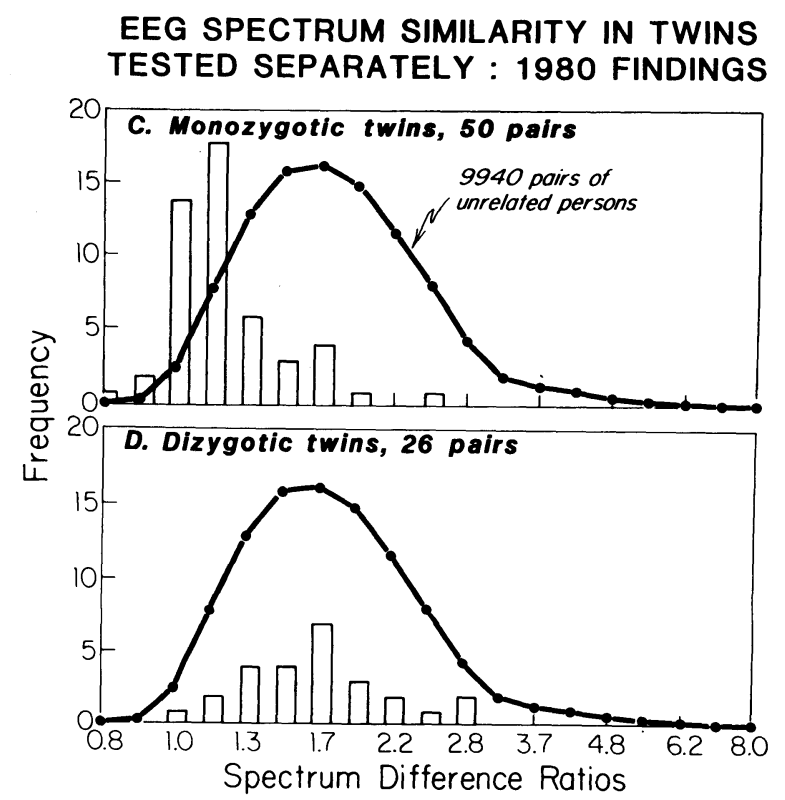

Figure 4. Results of the replication of the study shown in Figure 3. The findings are essentially identical. 
the within-pair similarity with respect to the various parameters that characterize an EEG spectrum. Four such parameters are defined in terms of the mean activity within each of the classical EEG frequency bands: delta $(.1$ to $2.9 \mathrm{~Hz})$, theta $(3.0$ to $7.9 \mathrm{~Hz})$, alpha $(8.0$ to $13.0 \mathrm{~Hz})$, and beta $(13.1$ to $20.0 \mathrm{~Hz})$. A fifth parameter, phi, is the median frequency of the alpha activity that appears as an elevation or "bump" in the midrange of the spectrum. Specifically, phi is the median frequency in a $3-\mathrm{Hz}$ band centered on the peak or highest point of the spectrum from 7.1 to $13.0 \mathrm{~Hz}$. Since some individuals display two clear peaks, the algorithm in such cases centered the $3-\mathrm{Hz}$ interval at the weighted midpoint between the two. For each of the 284 twins in the combined samples, these five parameters were computed for each of their four spectra. For the 1974 sample, these spectra were obtained from $\mathrm{Cz}$ at four times during the session; for the 1980 sample, spectra were obtained from $\mathrm{C} 3$ and $\mathrm{C} 4$ at the start and the end of the session. Averaging over the four spectra gave a relatively stable set of five parameters for each individual. A similar set of parameters, obtained like those from the 1980 sample, were obtained from a sample of 25 pairs of adult MZ twins who had been separated in infancy and reared apart (MZA twins). Parabolic regressions of each parameter on age were computed separately for male and female subjects. From the three regression coefficients, the computer could predict each subject's parameters based just on age and sex. The difference between the actual and the predicted parameter provided a value independent of age and sex for use in calculating within-pair correlations. Age- and sex-corrected intraclass correlations were computed for each parameter separately for the three twin types (MZA, MZT, and DZT) and are shown in Table 1.

It can be seen in Table 1 that all five parameters are strongly correlated within pairs of both groups of $\mathrm{MZ}$ twins. The $\mathrm{DZ}$ correlations were much smaller and, except for beta, were less than half as large as the corresponding $\mathrm{MZ}$ correlation. However, although the parameter means did not differ for MZs vs. DZs, the DZ total variance was smaller than the MZ variance in every case. As shown in column 4 of Table 1 , the ratio of $\mathrm{DZ}$ to $\mathrm{MZ}$ total variance ranged from only $52 \%$ for theta to $88 \%$ for beta. Since there is no reason to suppose that EEG parameters have less variance among $\mathrm{DZ}$ than among MZ twins in general, the difference observed here may be a result of the recruitment bias discussed by Lykken, Tellegen, and DeRubeis (1978), which attenuates between-pair variances in volunteer samples of $\mathrm{DZ}$ twins and thus results in underestimation of the true $\mathrm{DZ}$ correlation. By assuming that the total variance of an unbiased DZ sample should equal the total variance of the $\mathrm{MZ}$ sample, one can calculate "corrected" estimates of the true DZ intraclass correlation from:

$$
\hat{\mathbf{R}}_{\mathrm{dz}}=1-\frac{\mathbf{M S W}_{\mathrm{dz}}}{\mathbf{V}_{\mathrm{Tmz}}}
$$

These values are shown in column 5 of Table 1. For the parameters delta, theta, alpha, and beta, these corrected $\mathrm{DZ}$ correlations are, respectively, $36 \%$, $54 \%, 41 \%$, and $69 \%$ of the corresponding MZ correlations, as shown in column 6 . The mean MZ correlation for these four parameters is .805 , while the mean DZ correlation is .395, or about one-half of the $M Z$ value. For the parameter phi, alpha frequency, the corrected $\mathrm{DZ}$ correlation is only .13 , or about $16 \%$ of the $\mathrm{MZ}$ correlation of .81 .

\section{DISCUSSION}

This rather tortuous analysis suggests that the relative amount of activity in the four classical EEG frequency bands, which is strongly correlated within pairs of MZ twins raised either together or apart, may actually comport with the expectations derived from the standard polygenic additive model. It is

Table 1

Intraclass Correlations of Five Spectrum Parameters in Three Samples of Adult Twins Including 25 Pairs of Monozygotic Twins Separated as Infants and Reared Apart (MZA)

\begin{tabular}{|c|c|c|c|c|c|c|}
\hline & \multicolumn{3}{|c|}{ Intraclass Correlations } & \multicolumn{3}{|c|}{ Total Variance } \\
\hline & MZA & MZ* & $\mathrm{DZ} *$ & $\mathrm{DZ} / \mathrm{MZ}^{*}$ & $\mathrm{DZ}^{*}$ & $\mathrm{DZ} / \mathrm{MZ}+$ \\
\hline $\begin{array}{l}\text { N of Pairs } \\
\text { Delta } \\
\text { Theta } \\
\text { Alpha } \\
\text { Beta }\end{array}$ & $\begin{array}{l}25 \\
.90 \\
.76 \\
.93 \\
.61\end{array}$ & $\begin{array}{l}89 \\
.84 \\
.80 \\
.86 \\
.72\end{array}$ & $\begin{array}{l}53 \\
.26 \\
.04 \\
.13 \\
.37\end{array}$ & $\begin{array}{l}.71 \\
.52 \\
.61 \\
.88\end{array}$ & $\begin{array}{l}.30 \\
.43 \\
.35 \\
.50\end{array}$ & $\begin{array}{l}36 \\
54 \\
41 \\
69\end{array}$ \\
\hline Mean & .80 & .81 & .12 & .68 & .40 & 49 \\
\hline Phi & .89 & .81 & -.15 & .75 & .13 & 16 \\
\hline
\end{tabular}

Note-The total variance of all five spectra was smaller in the $D Z$ than in the $M Z$ sample (Column 4), which suggests that the DZ correlations (Column 3) may underestimate true values. Estimates of the "true" values (see text) are given in Column 5 . These estimates are expressed as a percentage of the corresponding $M Z$ correlations in Column $6 . \quad{ }^{*}$ Correlations and variances were computed separately for the 1974 and 1980 samples. The values entered in the table are averages weighted for sample size. † In percent. 
clear, however, that alpha frequency, phi, does not fit the standard model because, while the MZ correlation is large $(.81)$, the $\mathrm{DZ}$ correlation is not significantly larger than zero, even when "corrected" as described above. Alpha frequency, therefore, seems to behave like an emergenic trait, which implies that its value is determined by the interaction-rather than the simple summation-of several component traits, each of which is independently heritable. The fact that $M Z$ twins have similar alpha frequencies while DZ twins do not could, by itself, account for the findings with the spectrum difference ratio shown in Figures 3 and 4. Because the alpha "bump" is such a salient feature of most EEG spectra, if a pair of $\mathrm{DZ}$ twins differ significantly just in the median frequency of this "bump," this difference alone could yield a large r.m.s. difference between their spectra even if their other spectrum parameters were reasonably similar.

The fact that alpha frequency (at least) behaves like an emergenic trait is of particular interest because it is probable that some sort of resonance phenomenon is involved in the determination of alpha frequency and resonance is a paradigmatic example of an interactive or configural property. One can show the resonance features of EEG alpha by the type of experiment illustrated in Figure 5. This subject's normal eyes-closed EEG spectrum is shown at the top. Additional spectra were obtained while the subject watched a photostimulator flashing at a series of constant frequencies spanning the alpha band. Such photic driving produces a spike in the EEG spectrum at the driving frequency. As shown at the bottom of Figure 5, the height of this spike-the amplitude of the photic driving effect-increases as the driving frequency approaches that subject's natural alpha frequency. Thus, the alpha generator appears to resonate with the stimulus.

The nature and location of the alpha generator is at yet unknown, but the fact that it behaves like a resonant circuit may explain why the alpha frequency can be strongly correlated in $\mathrm{MZ}$ twins but not in $\mathrm{DZ}$ twins. If the various components of the alpha generator are each genetically determined, then $\mathrm{MZ}$ twins will be very similar in respect to each component and therefore also will have similar alpha frequencies. DZ twins, and other first-degree relatives, might be about half as similar as the $\mathrm{MZ}$ twins with respect to each component and yet be no more similar with respect to alpha frequency than random pairs of unrelated persons. This is because the resonant frequency of a system is a configural property, determined by some function of the product-rather than of the sum-of the relevant properties of the system components. This means that moderate differences in component properties, such as might be found in pairs of DZ twins, may be amplified into large dif-

\section{ILLUSTRATION OF RESONANCE}
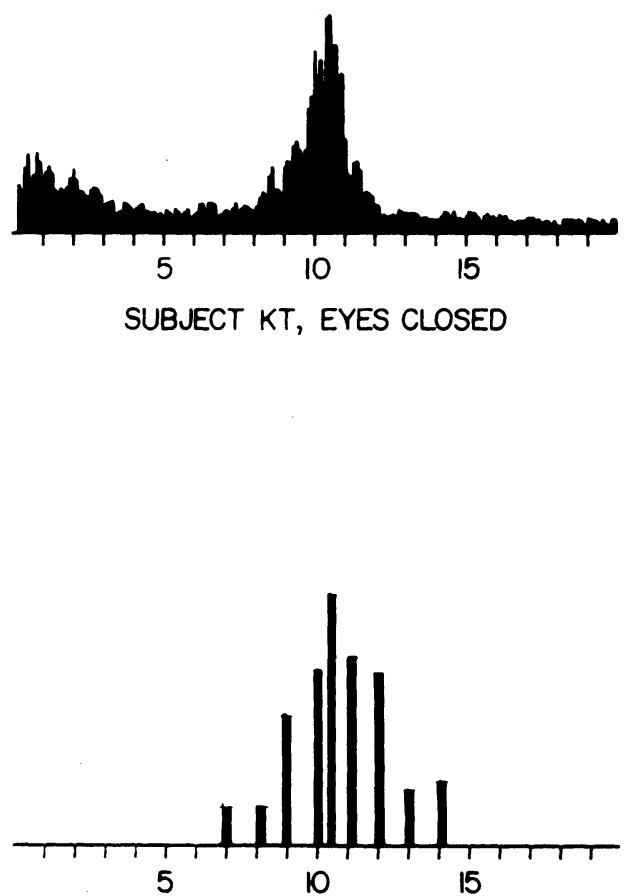

AMOUNT OF PHOTIC DRIVING, VARIOUS FREQUENCIES

Figure 5. An illustration of resonance in the EEG. When a subject views a light flashing at some frequency within the alpha range, a spike appears in the EEG spectrum at the stimulus frequency. The amount of this photic driving is maximum at the midfrequency of that subject's spontaneous alpha activity.

ferences in alpha frequencies when they are multiplied together.

\section{CONCLUSIONS}

These results confirm our original finding and appear to rule out an explanation based on a tendency for $\mathrm{DZ}$ twins to behave in a mutually divergent or complimentary fashion when examined together. Although MZ twins, whether reared together or apart, will usually produce strikingly similar EEG spectra when tested under similar conditions, the r.m.s. differences between the spectra of DZ twins are distributed like those between pairs of unrelated persons. We have provided somewhat equivocal evidence that certain spectrum parameters, viz., the relative amount of activity in the four classical EEG frequency bands, which are strongly correlated within pairs of $\mathrm{MZ}$ twins, may be moderately correlated in DZ twins, as would be predicted from the usual polygenic additive 
model. It is unequivocal, however, that the midfrequency of the EEG alpha activity, which is also strongly correlated in $\mathrm{MZ}$ twins, failed to show a significant intraclass correlation in our combined sample of 53 pairs of DZ twins. The response of the alpha generator to photic driving suggests that the generator has the properties of a resonant circuit. That the (unidentified) components of this resonant system are each strongly determined by the genome is indicated by the similarity of the alpha frequency within pairs of MZ twins. The lack of similarity of alpha frequency within pairs of $D Z$ twins might result from the fact that the resonant frequency of a system is a function of the product, rather than of the sum, of the relevant properties of the components of the system. We conclude, therefore, that the similarity of EEG spectra and, in particular, of the frequency of the alpha rhythm within pairs of MZ twins represent examples of "emergenesis," the interaction of multi- ple, independently inherited genotypic traits to produce a phenotype that is genetically determined but which is unlikely to show familial resemblance.

\section{REFERENCES}

LYKKEN, D. T. Statistical significance in psychological research. Psychological Bulletin, 1968, 70, 151-159.

LYKKEN, D. T. Psychometric applications of the EEG. In D. C. Fowles (Ed.), Clinical applications of psychophysiology. New York: Columbia University Press, 1975.

Lykken, D. T., Tellegen, A., \& Thorkelson, K. A. Genetic determination of EEG frequency spectra. Biological Psychology, 1974, 1, 245-259.

Lykken, D. T., Tellegen, A., \& DeRubeis, R. Volunteer bias in twin research: The rule of two-thirds. Social Biology, 1978, 25, 1-9.

MurPhy, E. A. Quantitative genetics: A critique. Social Biology, $1979,26,126-141$.

(Manuscript received December 18, 1981; accepted for publication February 10,1982.) 